QUADERNS DE FILOSOFIA VOL. IV NÚM. 2 (20I7): 35-58

eISSN: 234 I-3042 DOI: I 0.7203/QFIA.4.2.9482

Carla Isabel Velásouez

Universidad Autónoma de Barcelona

\title{
Replanteamiento de fondo en la enseñanza de la filosofía: problemas y apropiación
}

\author{
Deeply rethinking the teaching of philosophy: \\ problems and appropriation
}

Recibido: 1/1/17. Aceptado: 10/9/17

Resumen: ¿Para qué sirve la filosofía? es la pregunta que se hace desde fuera de la comunidad filosófica y cuya respuesta puede abrir puertas o poner serios obstáculos a su consolidación como asignatura. El permanente cuestionamiento externo por la "falta de utilidad" y la constante labor apologética que genera, parecen ser un indicio de que la filosofía requiere innovación en algunos aspectos de su enseñanza. A fin de contribuir con este esfuerzo es imprescindible identificar los problemas, y se determina que la falta de interacción con la ciencia puede ser uno de ellos. Se presentan dos ideas que se enlazan metodológicamente con este cambio de enfoque: una es poner el énfasis en los problemas filosóficos y no en autores; y la otra es facilitar que los estudiantes se apropien intelectualmente de la filosofía como elemento constitutivo de su pensamiento. Ambas ideas se implementarían sobre el trasfondo de establecer una relación estrecha con la ciencia, así como realizar cambios en la didáctica.

Abstract: How is philosophy useful? is the question posed from outside the philosophical community and whose answer can either open doors or become a serious obstacle to its consolidation as a subject. The constant external questioning regarding its "lack of usefulness", and the apologetic task it generates, seem to be an indication that philosophy requires innovation in some aspects of its teaching. In order to contribute to this effort it is essential to identify the problems, and it is 
determined that the lack of interaction with science may be one of them. Two ideas which are methodologically linked to this change of approach are presented: one is to underscore the philosophical problems and not the authors; and the other one is to enable the students to appropriate philosophy intellectually as a constitutive element of their thinking. Both ideas would be implemented on the basis of establishing a close relation with science, as well as making some changes in didactics.

Palabras clave: educación, innovación, filosofía, ciencia, problema, apropiación. Keywords: education, innovation, philosophy, science, problem, appropriation.

\section{INTRODUCCIÓN}

$\mathrm{E}$ N ESTE ARTículo se esbozarán dos ideas para innovar en la enseñanza de la filosofía. Tal innovación apuntaría a contribuir ante todo a que los estudiantes la reconozcan como valiosa y relevante, y que, por extensión, la comunidad educativa y la sociedad también la vean así. La primera idea consiste en enseñar enfatizando los problemas filosóficos y no los autores, lo cual no se debe interpretar en absoluto como una pretensión de eliminarlos, sino como no centrarse primordialmente en ellos. De esta manera la realidad, las ideas y el estudiante serán quienes ocupen el centro de importancia en el aprendizaje de la filosofía. Esta idea da paso a la segunda, que se trata de facilitar que los estudiantes se apropien la filosofía, es decir, que el "modo de ver" filosófico entre a formar parte del entramado constitutivo de su pensamiento. Para lograrlo puede usarse un método "socratizado" en el cual el docente guía a los estudiantes y les provee de las herramientas adecuadas con gran habilidad mayéutica, favoreciendo que piensen por sí mismos y se aproximen inicialmente a las respuestas. Este proceso, de hecho, los prepararía mejor para entender posteriormente a los autores.

Es necesario aclarar de entrada que no se aspira a que estas propuestas educativas sean las únicas válidas; de hecho, se asume que no existe solo una o unas que sean las aceptables y eliminen a las demás. Tampoco se busca argumentar en contra de otras posturas o compararse con ellas, sino más bien argüir a favor de una nueva que pueda aportar soluciones, porque se da por hecho que una pluralidad de aproximaciones es posible y es aplicable en diferentes instancias y momentos del proceso educativo. También es preciso explicar que para sustentar ambas ideas se tendrán en cuenta algunos aportes de los filósofos Bertrand Russell, Mario Bunge y José Ferrater Mora, quienes han tenido en común promover una relación muy estrecha entre filosofía y ciencia, la cual tendrá capital importancia en la argumentación, como se 
verá. Hechas estas aclaraciones, pasemos a contextualizar el problema del cual partiremos.

¿Para qué sirve la filosofía? Es una pregunta metafilosófica que todo filósofo en algún momento se formula y para la cual a veces no tiene una respuesta definitiva sino una idea provisional, como sucede también con la cuestión metafilosófica por excelencia: ¿qué es la filosofía? Muchos filósofos no podrían dar una definición exacta, o no querrían hacerlo por la complejidad del asunto, pero están familiarizados con este ambiente de incertidumbre que no les impide continuar filosofando. Sin embargo, por otro lado, visto desde fuera del mundo de la filosofía, hace falta responder satisfactoriamente al interrogante sobre cuál es su utilidad. Hay un número no desdeńable de actitudes de indiferencia y aversión hacia la filosofía que se ven entre algunos estudiantes, en la sociedad y en el gobierno, las cuales ponen la asignatura en un continuo entredicho que cíclicamente se intensifica. Podemos considerar seriamente la posibilidad de que esto sucede por no haberles dado hasta ahora, o al menos no a todos, una réplica convincente a la cuestión de la utilidad del saber filosófico. Algunos filósofos tienen una postura bastante radical, opuesta por principio a la "utilidad" de la filosofía. Sostienen que es valiosa en sí misma y no porque le sirva a otra cosa; que se busca por ser lo que es, como el arte, y no por su valor práctico; que no se subordina a nada; que no es una "herramienta" para "usar". Parece haber implícita en esta postura una concepción peyorativa de la "utilidad", que podría deberse más a un problema terminológico que de sustancia. Por otra parte, podría decirse que la mayoría de filósofos sí aceptan la "utilidad", afirmando que sirve para desarrollar el pensamiento crítico, aprender a analizar, pensar de manera lógica, cuestionar supuestos, reflexionar sobre cómo actuar, entre otros. Nos ubicaremos en este último tipo de respuesta. En cualquier caso, decir que es "útil" o que "sirve" puede ser asumido de diversas maneras, por ejemplo desde distintos modos de tratar los problemas. A propósito, queriendo responder al interrogante de para qué sirven los filósofos dijo José Ferrater Mora:

¿Para qué preguntarse (o preguntarnos) para qué servimos?: servimos para todo. Nadie nos puede batir en la variedad y universalidad de intereses. Lo malo es que los modos como los filósofos suelen tratar estos, y muchísimos otros, problemas, no parece convencer a mucha gente. Para empezar, los filósofos suelen caer víctimas de una de estas dos opuestas tendencias (y a veces ambas): o se pierden en nebulosas especulaciones... o se enzarzan en menudos y detalladísimos análisis que no conducen a ninguna parte, salvo a reconocer que de aquello de que en cada caso se habla hay siempre muchísimo más que hablar. La cuestión “¿Para qué sirven los filósofos?” persiste, y con ella la tentación de concluir que no sirven para nada (Ferrater Mora i 994, 64). 
Los dos modos de tratar problemas que mencionó aquí Ferrater son más característicos de una filosofía que se considera útil pero sin salir de sí misma, situación que puede acabar por ser muy limitante para quien no quiera confinarse dentro de los límites de su discurso. Esta actitud de replegarse sobre sí obedece en muchas ocasiones a diferenciar tajantemente el discurso filosófico del de la ciencia, lo cual lleva a que el filósofo se instale en "la torre de marfil". Definiremos a continuación los matices del término "útil" como lo entenderemos aquí, siguiendo el tratamiento que Bertrand Russell hace de la cuestión en diversos lugares. Veamos.

En On Education (2003) Russell habló de la controversia moderna acerca de si la educación debe ser "útil" en vez de "ornamental"; por ejemplo, de si se deben aprender principalmente los clásicos o ciencia. Concluyó que esta oposición no es real si se considera la definición amplia de "útil": "En el sentido más amplio y más correcto de la palabra, una actividad es 'útil' cuando tiene buenos resultados... Cuando así se define útil, no hay duda sobre si la educación debería ser útil. Por supuesto que debería, ya que el proceso de educación es un medio para un fin, no un fin en sí mismo" (Russell 2003, 17-8). ${ }^{1}$ No parece necesario decirlo porque es evidente, pero vale la pena explicitar que Russell sabía que el origen de la polémica era el sentido que "útil" tenía para muchos, y prosiguió: "Pero eso no es lo que los defensores de la utilidad en la educación tienen en mente. Lo que ellos están instando es que el resultado de la educación debe ser útil: hablando sin rodeos, ellos dirían que un hombre educado es un hombre que sabe cómo hacer máquinas" (Russell 2003, 18). ${ }^{2} \mathrm{Al}$ continuar con el tratamiento detallado de este asunto, el autor llegó a la conclusión, entre otras, de que ambos tipos de educación son beneficiosos, porque sin la ciencia el mundo de hoy no habría sido posible y sin lo humanístico faltaría estímulo para la imaginación, la cual es eso que permite vislumbrar lo que el mundo puede llegar a ser y sin la cual el progreso sería mecánico y trivial. Cabe tener en cuenta que el autor afirmaba que la ciencia también estimula la imaginación (Russell 2003, 14-32). En la misma línea dijo en "Science and Education" (Russell 2009, 597-601), que la enseñanza debe no solamente procurar que los estudiantes adquieran habilidades científicas, sino que además debe estimular las emociones positivas, de forma que las negativas no sean el motor para la acción, la cual a su vez involucra la ejecución de tales habilidades.

1 "In the widest and most correct sense of the Word, an activity is 'useful' when it has good results... When useful is defined this way, there is no question whether education should be useful. Of course it should, since the process of educating is a means to an end, not an end in itself” (Russell 2003, 17-8).

2 "But that is not what the advocates of utility in education have in mind. What they are urging is that the result of education should be useful: put crudely, they would say that an educated man is a man who knows how to make machines" (Russell 2003, 18). 
El autor consideraba que es obvio que la ciencia por sí sola no puede proporcionar las actitudes positivas que el mundo necesita. También dijo algo similar en "Education's Place in a New Age" (Russell 1953), donde trató sobre la aparente dicotomía entre dos objetivos de la educación, a saber, dar habilidad o dar sabiduría. Afirmó que la enseñanza de únicamente las habilidades científicas quedaría incompleta y podría llegar a ser incluso bastante destructiva, ya que la ciencia puede proveer los medios para un fin pero no puede ayudar a decidir cuáles fines se deben perseguir. En otros términos, puede brindar las herramientas para alcanzar ya sea el exterminio o la prosperidad de la humanidad, mas en última instancia no puede indicar cuál fin es más deseable. En "The Divorce of Science and Culture", Russell (1958) habló también de la tendencia a oponer las humanidades como "excelencia intrínseca", a la ciencia como "eficiencia causal", oposición que consideraba errónea:

En mi propio país, y en un grado menor en otros países de occidente, la "cultura" es vista principalmente, en un desafortunado empobrecimiento de la tradición renacentista, como algo relacionado primordialmente con la literatura, la historia y el arte. Un hombre no es considerado inculto si no sabe nada sobre las contribuciones de Galileo, Descartes y sus sucesores... Hay dos formas muy diferentes de evaluar cualquier logro humano: se puede evaluar según lo que se considere como su excelencia intríseca; o se puede evaluar según su eficiencia causal en transformar la vida humana y las instituciones humanas. No estoy sugiriendo que una de estas formas de evaluar sea preferible a la otra. Solo me interesa seńalar que ellas proporcionan escalas de importancia muy diferentes. Si Homero y Esquilo no hubieran existido, si Dante y Shakespeare no hubieran escrito ni una línea, si Bach y Beethoven hubieran estado en silencio, la vida diaria de la mayoría de la gente a día de hoy habría sido prácticamente lo que es. Pero si Pitágoras y Galileo y James Watt no hubieran existido, la vida diaria, no solo de los europeos occidentales y de los americanos, sino también la de los campesinos indios, rusos y chinos, sería profundamente diferente de la que es (RUSSELL I958)

3 "In my own country, and to a lesser degree in other countries of the West, 'culture' is viewed mainly, by an unfortunate impoverishment of the Renaissance tradition, as something concerned primarily with literature, history and art. A man is not considered uneducated if he knows nothing of the contributions of Galileo, Descartes and their successors...There are two very different ways of estimating any human achievement: you may estimate it by what you consider its intrinsic excellence; or you may estimate it by its causal efficiency in transforming human life and human institutions. I am not suggesting that one of these ways of estimating is preferable to the other. I am only concerned to point out that they give very different scales of importance. If Homer and Aeschylus had not existed, if Dante and Shakespeare had not written a line, if Bach and Beethoven had been silent, the daily life of most people in the present day would have been much what it is. But if Pythagoras and Galileo 
En conclusión, de acuerdo con Russell la educación debería integrar humanidades y ciencia, y por tanto, filosofía y ciencia, dándole importancia a ambas. Las dicotomías que se han visto, útil/ornamental, ciencia/emoción, habilidad/sabiduría, excelencia/eficiencia no son irresolubles porque se refieren a diferentes aspectos del conocimiento y del desarrollo humano. Una de las razones fundamentales para integrar tales aspectos en el proceso educativo es que trascienden de lo individual a lo social, como lo analizó el autor en Education and the Social Order (1932), donde presentó casos en los que se intenta determinar el papel de la educación desde extremos opuestos: individuo/ciudadano, individuo/comunidad, saber/sentir particular. Afirmó que hay diferencias pero no antítesis: "Los elementos de conocimiento y emoción en el individuo perfecto como lo hemos estado representando no son esencialmente sociales. Es solo a través de la voluntad y a través del ejercicio del poder que el individuo a quien hemos estado imaginando se convierte en un miembro efectivo de la comunidad" (Russell 1932, 12). ${ }^{4}$ De esto se puede deducir que para ser miembro de una comunidad mejor, el ser humano debe verse a sí mismo no exclusivamente como producto de ésta, sino también como causa del cambio tanto individual como social. Esta visión le da una perspectiva de creación y de responsabilidad desde sí mismo, que se deriva de "los elementos de conocimiento y emoción" cultivados en la educación y cuyos efectos se materializarán de una u otra forma en la sociedad. Las "utilidades" de las humanidades según Russell consideradas hasta el momento son: estimular la imaginación, crear actitudes constructivas y ayudar a decidir qué fines se deben perseguir como individuo y como sociedad. Sobra decir que Russell tuvo muchas más ideas sobre educación que las antes mencionadas (Russell I954; Hare 1987).

Después de haber precisado el sentido amplio de "utilidad" que adoptaremos aquí, podemos concluir que no vale la pena prescindir de emplear el adjetivo "útil" con referencia a la filosofía, pero que adquiere un significado diferente según sea la interacción de ésta con la ciencia. Una actitud anticientífica hace que la filosofía se relegue a sí misma frente al resto de disciplinas, lo cual no es necesario ni deseable según nuestra visión. Si se habla de que la filosofía es "útil" para pensar, pero se insiste en crear discordia con la ciencia y la tecnología, consideramos que se estaría ofreciendo una "utilidad" no solo muy

and James Watt had not existed, the daily life, not only of Western Europeans and Americans but of Indian, Russian and Chinese peasants, would be profoundly different from what it is" (RusSELL I958).

4 "The elements of knowledge and emotion in the perfect individual as we have been portraying him are not essentially social. It is only through the will and through the exercise of power that the individual whom we have been imagining becomes an effective member of the community" (RuSSELl I932, 12). 
limitada, sino que también podría conducir al error. Bertrand Russell opinaba sobre los intelectuales que no se abren al mundo:

En el presente, la técnica científica avanza como un ejército de tanques que han perdido sus conductores, ciegamente, sin piedad, sin objetivo ni propósito. Esto ocurre en gran medida porque los hombres que se interesan por los valores humanos y por hacer que la vida valga la pena ser vivida, están todavía viviendo en la imaginación en el viejo mundo preindustrial, el mundo que ha sido hecho familiar y cómodo por la literatura de Grecia y los logros preindustriales de los poetas y artistas y compositores cuyo trabajo nosotros con toda razón admiramos (RUSSELL 1958)

Si la filosofía se enseña como una especialidad puramente metafilosófica y textualista, y desligada del acontecer mundano, es entendible que acabe por quedarse aislada académica y socialmente. A propósito, el filósofo Manuel Sacristán (1968) propuso en su famoso panfleto Sobre el lugar de la filosofía en los estudios superiores eliminar la filosofía de las universidades y de la enseñanza media, ya que la autonomía hermética de la filosofía profesional, según él, desfiguraba las verdaderas cuestiones filosóficas que no son puramente metafilosóficas o de interpretación filosófica. Decía que la filosofía no tenía un carácter sustantivo sino adjetivo, y por tanto no aportaba nada al progreso científico por sí sola. Por ese motivo, sugirió que las secciones de filosofía deberían ser reemplazadas por un solo instituto donde se hiciera una reflexión filosófica en conexión con todos los saberes y donde participaran miembros de todos los campos del conocimiento científico (SACRISTÁN 1968). Ferrater Mora comentó esta propuesta y concluyó que no estaba de acuerdo con Sacristán en el extremo de suprimir la filosofía, pero dijo que sin embargo: "Sacristán da en el clavo al poner de relieve que la filosofía funcionaría mejor si dejara de ser una disciplina 'especializada' y se abriera más a otros campos del saber" (Ferrater i 982, 190). Habría que comenzar el proceso de innovación, entonces, por evitar esta "desconexión" y "aislacionismo". Es entendible que muchos filósofos no darían un paso hacia un replanteamiento de fondo en la enseñanza de la filosofía de la manera que aquí se propone, bien porque no acepten nuestro sentido de "utilidad", o porque

\footnotetext{
5 "At present, scientific technique advances like an army of tanks that have lost their drivers, blindly, ruthlessly, without goal or purpose. This is largely because the men who are concerned with human values and with making life worthy to be lived, are still living in imagination in the old pre-industrial world, the world that has been made familiar and comfortable by the literature of Greece and the pre-industrial achievements of the poets and artists and composers whose work we rightly admire" (Russell I958).
} 
no estimen que sea necesario entreverarse con la ciencia, no viendo un mal en el aislamiento. Se admite que nuestra actitud, aunque abierta, no podría contribuir mucho a ideas diametralmente opuestas a la ciencia, como que la filosofía ha muerto, que la realidad ocurre solo en el lenguaje o en los símbolos, que todo es relativo a la cultura, que la ciencia y la tecnología "cosifican" las cosas, o que no hay distinción entre teoría o textos y realidad, que la verdad no existe ni por aproximación, que no se puede llegar al conocimiento, por mencionar algunas. Estas concepciones y otras similares provienen de filosofías con tendencia a un lenguaje obscurantista, que se expresan en textos de carácter exclusivamente especulativo e inentendibles. Si así fuera la filosofía que se habría de propugnar, no entenderíamos para qué se enseñaría ni por qué valdría la pena aprenderla; en consecuencia, no dirigiremos nuestro esfuerzo en esa dirección. Nos encaminamos a que la filosofía se exprese de modo que sea "traducible" a otros niveles (ámbitos, disciplinas, medios, etc.) sin perder el rigor y así evitar dos riesgos: "degradar la filosofía tratando a toda costa de colocarla al alcance de cualquier público y asfixiarla manteniéndola perfectamente incomunicada" (Ferrater i 982, 187). Partiendo de que es posible "traducir" los lenguajes, el deseo de hacerse entendible y de intentar entender otras áreas del conocimiento, sería promovido como una actitud intelectual constructiva de la filosofía, en consonancia con lo que se ha visto que dijo Russell sobre la actitud de la educación, que debe ser optimista, imaginativa e inclinada hacia fines constructivos: "La educación es esencialmente constructiva y requiere alguna concepción positiva de lo que constituye una buena vida" (Russell 2009, 380); ${ }^{6}$ y en concordancia: "Todo lo que ha hecho grandioso al hombre ha brotado del intento de asegurar lo que es bueno, no de la lucha por evitar lo que se pensaba que era malo. Como la educación moderna es tan raramente inspirada por una gran esperanza, es muy raro que alcance un gran resultado" (Russell i 9i 6). ${ }^{7}$

La disociación entre filosofía y ciencia, no solo no es conveniente por motivos pragmáticos, sino que ante todo no es correcta porque, según nuestra concepción, en su raíz ontológica la realidad no sucede en compartimentos o casillas sino que es un sistema de niveles continuos, con problemas sistémicos y que requiere, por consiguiente, soluciones sistémicas. De acuerdo con las teorías ontológicas de Mario Bunge (1977; 1979) y de José Ferrater Mora (1998), la realidad es una, pero puede ser estudiada desde sus distintos

6 "Education is essentially constructive, and requires some positive conception of what constitutes a good life" (Russell 2009, 380).

7 "All that has made man great has sprung from the attempt to secure what is good, not from the struggle to avert what was thought evil. It's because modern education is so seldom inspired by a great hope that it so seldom achieves a great result" (RusselL I9I6). 
niveles, tal como ocurre con el ser humano, que es uno y opera como un sistema a la vez físico, químico, biológico, social, cultural y técnico; al estudiarlo como sujeto ético, por ejemplo, se le estudia normalmente desde el nivel cultural, pero sería un estudio incompleto si no se tuvieran en cuenta los componentes biológicos y sociales que lo constituyen en un nivel más fundamental, que conforman su entorno y que son estudiados por las ciencias. En concordancia con la unidad ontológica de la realidad, la filosofía debe abrirse a una relación epistemológica con la ciencia y visceversa, pero en ningún caso se afirma que la una reemplace a la otra. Ferrater Mora afirmó en varias obras que la relación con la ciencia por parte de la filosofía es triple: analítica, crítica y de revision conceptual (Ferrater, 1974; 1976; 1982; I998). Esta relación es posible precisamente porque los "lenguajes" de ambas no son totalmente intraducibles. Mario Bunge, uno de los filósofos de la ciencia más importantes del siglo xx, y además físico (véase, por ejemplo, BungE I967A; 2003B; 20 I 5), es conocido por sus aportes bidireccionales. Ha contribuido a la epistemología de la ciencia, a la ética de la ciencia y a la fundamentación y el fortalecimiento de las ciencias sociales, entre otros, trabajando en estrecho contacto con expertos de las ciencias por las que se ha interesado. Uno de sus aportes más recientes es Doing Science in the Light of Philosophy (2017), donde promueve que los científicos le den una perspectiva filosófica a la investigación, porque como él ha venido diciendo: "la ciencia, la técnica y la filosofía no son departamentos aislados entre sí, sino componentes de un sistema. En particular, muestran que la filosofía adoptada por una rama de la ciencia o de la técnica puede estimularla o inhibirla” (Bunge 1998, 151). En este sentido, la filosofía es útil porque capacita, entre otras cosas, para tener una visión crítica general transdisciplinaria (inter y multi), sin confundir el conocimiento, que es resultado de un proceso cognitivo, con la realidad, que es lo que es. Esta visión es una herramienta para saber enfrentarse de forma sistémica a los problemas con objetividad.

En conclusión, desconocer la interdependencia y, más aún, crear antagonismo entre el saber filosófico y el científico sería un error, que en efecto nunca ha tenido como resultado los grandes logros culturales de la humanidad según Bertrand Russell, quien como uno de los grandes filósofos y matemáticos del siglo $\mathrm{xx}$, dijo con conocimiento de causa:

La educación que Platón diseñó no era en su día lo que ahora se llamaría "cultural"... Lo que Platón principalmente deseaba que se enseñara en su academia era primero matemáticas y astronomía y, luego, filosofía. La filosofía habría de tener una inspiración científica con un toque de misticismo órfico... Las necesidades del mundo moderno han traido un conflicto, el cual creo que puede 
ser evitado, entre asuntos científicos y los llamados "culturales". Estos últimos representan la tradición y todavía tienen, en mi país, una cierta preeminencia esnobista. La ignorancia cultural, más allá de cierto punto, es despreciada. La ignorancia científica, por completa que sea, no lo es. No creo que la division entre la educación cultural y la científica debería estar ni de cerca tan definida como ha tendido a volverse (Russell I960, 15)

\section{Y en otro lugar afirmó:}

La separación entre ciencia y “cultura” es un fenómeno moderno. Platón y Aristóteles tenían un profundo respeto por lo que se conocía como ciencia en su día. El renacimiento estaba tan interesado por revivir la ciencia como por el arte y la literatura. Leonardo da Vinci dedicó más energías a la ciencia que a pintar (Russell I958)9

Según lo anterior, es irónico que hoy haya que "tender puentes" entre filosofía y ciencia, cuyo vínculo se da en muchos sentidos, entre ellos el metodológico. Veamos.

\section{Problemas}

El "problema” es el primer aspecto metodológico en el que coinciden la filosofía y la ciencia, es el punto de partida de sus indagaciones y ambas lo estudian críticamente. Sobre la importancia del "problema” dice Mario Bunge en la mencionada obra Doing Science in the Light of Philosophy:

Dedicarse a cualquier tipo de investigación es trabajar sobre un problema o grupo de problemas de algún tipo — cognitivo, tecnológico, social, artístico o moral. Imitando el evangelio de Juan, podemos decir que en el principio era el

8 "The education which Plato designed was not in his day what would be now called 'cultural'... What Plato mainly wished his Academy to teach was, first, mathematics and astronomy, and, then, philosophy. The philosophy was to have a scientific inspiration with a tincture of Orphic mysticism... The needs of the modern world have brought a conflict, which I think could be avoided, between scientific subjects and those that are called 'cultural'. The latter represent tradition and still have, in my country, a certain snobbish pre-eminence. Cultural ignorance, beyond a point, is despised. Scientific ignorance, however complete, is not. I do not think, myself, that the division between cultural and scientific education should be nearly as definite as it has tended to become" (Russell i960, 15).

9 "The separation of science from 'culture' is a modern phenomenon. Plato and Aristotle had a profound respect for what was known of science in their day. The Renaissance was as much concerned with the revival of science as with art and literature. Leonardo da Vinci devoted more of his energies to science than to painting" (Russell I958). 
problema. Entonces, quienes quieran empezar a hacer ciencia deben encontrar o inventarse un problema sobre el cual trabajar, así como un mentor que esté dispuesto a guiarlos (BUNGE 2017, 1) $)^{10}$

Según la definición de Ferrater Mora (1994, 2009), un problema es "una cuestión que se trata de aclarar o resolver", precisando que todo problema es implícita o explícitamente una pregunta, pero que no toda pregunta es un problema. Según la definición de Bunge (2000, I49) "el término 'problema' designa una dificultad que no puede resolverse automáticamente, sino que requiere una investigación, conceptual o empírica", y en otro lugar dice que es "un vacío de conocimiento que se juzga digno de ser llenado"11 (BUnGe 2003A, 227). Bunge hace una primera división dentro del conjunto de los problemas entre conceptuales y empíricos: "Mientras que los primeros requieren solo herramientas conceptuales, los últimos también requieren investigación empírica" ${ }^{12}$ (BUNGE 2003A, 227). Esta diferenciación ya evita múltiples fallos en filosofía, puesto que advierte, por ejemplo, que no se puede resolver un problema que necesita contrastación experimental desde un discurso puramente conceptual. Un caso de error sería no tener en cuenta los hallazgos de la psicología y la neurociencia cognitiva al tratar los problemas de la filosofía de la mente. En definitiva, elegir el problema y plantearlo correctamente es el primer paso importante de cualquier investigación. Por desgracia, a pesar de la preponderancia del problema, la educación se centra más en los datos que en lo buscado y en las respuestas a preguntas ya hechas con soluciones ya resueltas que a plantear los problemas. $\mathrm{Al}$ respecto dice Mario Bunge:

Una política educativa no dogmática, que estuviera de acuerdo con el espíritu de la ciencia y no nos empujara a entablar una carrera de competición contra las máquinas de calcular, estimularía el proceso de maduración de una actitud inquisitiva y de los métodos y teorías de más potencia: sería una pedagogía centrada en el problema, y entrenaría así al hombre a compensar la rápida decadencia de la opinión recibida (BUNGE 2000, 185)

10 "To engage in research of any kind is to work on a problem or a cluster of problems of some kind-cognitive, technological, social, artistic, or moral. In imitation of John's gospel, we may say that in the beginning was the problem. So, those wishing to start doing science must find or invent a problem to work on, as well as a mentor willing to guide them" (BUNGE 2017, 1).

11 "A gap in knowledge judged worthy of being filled" (BUNGE 2003, 227).

12 "A first partition of the set of problems is into conceptual and empirical. Whereas the former require only conceptual tools, the latter also demand empirical investigation" (BUNGE 2003, 227). 
Asumiremos, entonces, la preeminencia del problema en el conocimiento en general y en la filosofía en particular. Bunge dedica un apartado al "problema" en su tratado sobre filosofía de la ciencia, Scientific Research (1967в), traducido al castellano como La investigación cientifica (2000), presentando una lógica erotética, específica para aplicar a la forma, el contexto y el contenido de los problemas. Dentro del mencionado apartado se encuentra un tratamiento particular de los problemas filosóficos. Bunge establece allí una distinción, que no es de tipo valorativo, entre dos clases de filósofos que puede haber: por un lado, los que estudian problemas filosóficos y se interesan primordialmente por las ideas, llevando a cabo la actividad filosófica primaria; $y$, por otro lado, los que estudian lo que han dicho los que estudian problemas filosóficos. Estos últimos no se centran en los problemas, sino en las soluciones y se interesan principalmente no tanto por las ideas en sí, sino por su expresión y su contexto, dedicando su labor a comentar, explicar, enseñar, etc., un conjunto de doctrinas y opiniones, en general, lo que hacen los primeros. Bunge afirma que:

...las dos clases de filósofos son necesarias para que viva la filosofía, pero el progreso filosófico, igual que el de la ciencia, exige comprender claramente que (i) la investigación original consiste en descubrir, inventar, disolver y resolver problemas — a poder ser profundos y fecundos-, y (ii) que la investigación original es imprescindible para mantener una disciplina en vida (BUNGE 2000, 187)

Esta distinción no implica que los dos aspectos no puedan darse en un solo filósofo, pueden ocurrir en diferentes grados y en distintos momentos. Cada filósofo tiende más o menos hacia cada uno de los dos extremos. Dedicarse a ser filósofo del segundo tipo por elección propia es algo absolutamente normal, todo filósofo lo tiene que ser en cierta medida, pero lo preocupante es que muchos no escogen limitarse a permanecer ahí; se quedan anquilosados porque determinan que no alcanzarían a pertenecer al primer grupo, reservado para los grandes maestros o genios, o porque determinan que no pueden aspirar a pertenecer a ese grupo hasta que no dominen la totalidad de la tradición de lo que los otros han hecho, en cuyo estudio desbordante se quedan para siempre encallados. Desafortunadamente, estas autolimitaciones se dan por hecho o se aprenden y, asimismo, se transmiten de forma implícita o explícita, acabando por entorpecer la iniciativa de aproximarse de manera más directa, decidida y proactiva a los problemas.

Mario Bunge clasifica los problemas filosóficos en tres tipos: de lógica (de forma); de epistemología (de conocimiento); o de ontología (del ser). Tienen en común ser genéricos y conceptuales, por lo cual su formulación no contiene 
datos empíricos, pero en determinados casos presuponen un cuerpo de ciencia factual y en ese punto puede haber una interacción con la ciencia. Los problemas históricos, lingüísticos, psicológicos, etc., pueden servir para aclarar problemas filosóficos o como antesala a ellos (propedéutica), mas no son filosóficos en sí. Los grandes problemas de la filosofía abarcan demasiado pero pueden ser abordados formulando unos de menor alcance. Es el caso de ¿qué es el ser?, problema de enorme envergadura al cual nos podemos aproximar por medio de interrogantes más simples, por ejemplo sobre los tipos de existencia. Podríamos preguntar: ¿Qué es una cosa?, ¿Qué tipos de cosas existen?, ¿Una forma, una relación, una clase, un conjunto, una propiedad, un sistema o un proceso son cosas? O de manera menos formal: ¿Qué clase de objetos son un número, un símbolo, un fantasma, un libro, el dinero, una teoría, un grupo social, una galaxia, el universo? ¿En qué se diferencian?, etc. Es necesario entonces distinguir el alcance de los problemas y empezar a tratarlos ajustándose a las condiciones. Una forma de suscitar los grandes problemas puede ser empezar por considerar algunos que sean concretos y posteriormente ocuparse de los fundamentos teóricos, como dice Mario Bunge al hablar de cómo motivar a los estudiantes, citando el que fue su primer libro, Temas de educación popular (1943):

Para ello es preciso no empezar por mostrarle la belleza de los teoremas, sino la belleza de su utilidad práctica... Por esto no debe enseñarse la matemática aislada de la técnica, sino en función de ella. O sea, ... partir del problema concreto, mostrando entonces el método para resolverlo: así surgirá el teorema de la tentativa de resolver un problema concreto (BUNGE 2006, 89)

Una manera de generar problemas es buscar relaciones entre problemas filosóficos y científicos, cosa que sería sin duda provechosa para los estudiantes, quienes han estado adquiriendo conocimientos científicos desde temprana edad, aprendiendo desde pequeños que son muy valiosos y sin haber visto ningún choque entre ellos y las humanidades (lengua, literatura, sociales, historia, etc.). De hecho, si se les hicieran preguntas filosóficas a los estudiantes, sin haberles enseñado aún nada de filosofía como asignatura, probablemente la mayoría se valdrían de la ciencia que han estudiado a lo largo de su formación para intentar resolverlas y formar su improvisada "argumentación filosófica". Para enseñar filosofía se podría tomar, por poner un caso, un problema de biología y analizarlo en todos o varios de sus aspectos filosóficos:

Quien enfoque la biología desde la filosofía advierte que esa ciencia suscita problemas filosóficos de variada índole: ontológicos, como el de la naturaleza de la vida; gnoseológicos, como el de las peculiaridades de la biología respecto a la 
química; lógicos, como la estructura de los argumentos reduccionistas; semánticos, como el significado de términos tales como "información”, "programa genético" y "molde"; metodológicos, como el de la naturaleza de los indicadores que hacen puente entre los conceptos teóricos y los empíricos; y éticos, como los de la clonación de humanos y el uso terapéutico de células pluripotentes extraídas de embriones humanos (Bunge 2006, 29)

De todas maneras, continuará latente la posibilidad de ver la filosofía como algo demasiado abstracto. Ferrater Mora queriendo ejemplificar el caso de cómo mostrar a los estudiantes la utilidad de un conocimiento que en primera instancia parece inaplicable, puso el caso de enseñar sobre la guerra de Troya, previendo la réplica de muchos estudiantes, a saber: “¿qué nos importa la guerra de Troya?". Dijo el autor que: "Esta es la pregunta —o más bien, el grito de combate- que resume la oposición de muchos estudiantes al cultivo de estudios de supuesta escasa, o nula, significación para nuestra época" (Ferrater 1986, 114). Ferrater concluyó que ya es de por sí valioso el simple hecho de haber suscitado la pregunta de si nos importa o no tal guerra. No obstante, previó una segunda reacción:

Parece que solo queda por lanzar un último exabrupto: “¿Y qué importa que nos importe la guerra de Troya?”. En realidad, nada importa nada o, lo que viene a ser lo mismo, no puede justificarse absolutamente la importancia de nada. Si algo es importante, lo es en vista de alguna otra cosa, cuya importancia es relativa a otra, y así sucesivamente. No hay "importancias" absolutas, a menos de considerar que lo importante somos "nosotros". Pero, ¿quiénes somos "nosotros"? Somos unos a quienes no nos importa la guerra de Troya y otros a quienes nos importa grandemente: ¿van, pues, unos a contar más que otros? (FERRATER I986, 117)

Como se ha ido bosquejando, se estima que una forma de hacer más plausible el valor del conocimiento en cuestión es poner al estudiante en el centro del problema, como partícipe intelectual directo y no solo como recipiente pasivo de información y datos. Consideramos que hay que involucrarlo desde la reconstrucción del problema mismo y que es posible que opere de una forma autónoma y a la vez dirigida, la cual no consiste en comenzar de cero ni en dedicarse a decir la opinión personal sin filtros. Con el fin de llevar a cabo este proceso, comenzar por mencionar a los grandes autores sería contraproducente, puesto que, aunque no sea nuestra intención, el estudiante sin percatarse asume que "la filosofía" son unas teorías o ideas que conciben "los filósofos" y se asientan inadvertidamente en el lugar del espectador. Por lo tanto, sin desconocer que las grandes figuras del pensamiento son ineludi- 
bles para la disciplina filosófica, cuyo carácter histórico implica que heredamos tradiciones que no se dan en un contexto vacío, hemos de asegurarnos que su presencia no sea "aplastante": ¿podríamos comenzar a tratar un problema sin mencionar inicialmente el nombre de un autor ni de un lugar de modo que sea el mismo problema el que tenga preeminencia? Seguramente podríamos hacerlo, tal mención se puede como mínimo posponer, de forma semejante a como es posible enseñar física sin mencionar el nombre de Newton o de Einstein, Inglaterra o Alemania. Cabe reiterar que esto no quiere decir que no se deban mencionar nunca los nombres, ni que no reconozcamos los logros de las personas que han ido elaborando la tradición. El objetivo no es minimizar los méritos de los autores, sino no perder de vista que la realidad y las ideas, precisamente lo que esos mismos autores buscaban, son lo importante y lo que se debería destacar. Bertrand Russell dijo sobre Aristóteles algo que ejemplifica lo que pasa cuando se pone al autor por encima de las ideas:

Al leer a cualquier filósofo importante, pero sobre todo a Aristóteles, es necesario estudiarlo de dos formas: con referencia a sus predecesores y con referencia a sus sucesores. En el primer aspecto, los méritos de Aristóteles son enormes; en el último, sus deméritos son igualmente enormes. Por sus deméritos, sin embargo, sus sucesores son más responsables que él. Él vino al final del periodo creativo del pensamiento griego y después de su muerte pasaron dos mil años antes de que el mundo produjera algún filósofo que pudiera ser considerado como aproximadamente su igual. Hacia finales de este largo periodo su autoridad se había vuelto casi tan incuestionable como la de la iglesia y en la ciencia, así como en la filosofía, se había vuelto un grave obstáculo para el progreso (RUSSELL 2000, 173) ${ }^{13}$

En conclusión, comenzar a aprender filosofía por problemas y no por autores podría ser mucho más significativo para combatir de entrada la pasividad intelectual y que los estudiates desarrollen el ingenio y la capacidad de problematizar; y sería aún más enriquecedor si analizaran filosóficamente lo que están viendo en otras asignaturas y así ejercitar la integración bidireccional

13 "In reading any important philosopher, but most of all in reading Aristotle, it is necessary to study him in two ways: with reference to his predecessors, and with reference to his successors. In the former aspect, Aristotle's merits are enormous; in the latter, his demerits are equally enormous. For his demerits, however, his successors are more responsible than he is. He came at the end of the creative period of Greek thought, and after his death it was two thousand years before the world produced any philosopher who could be regarded as approximately his equal. Towards the end of this long period his authority had become almost unquestionable as that of the Church, and in science, as well as in philosophy, had become a serious obstacle to progress" (Russell 2000, 173). 
de la filosofía con la ciencia, cimentando de paso una sólida plataforma para los científicos del futuro. Veamos cómo podría ser ese proceso.

\section{Apropiación}

En España, a dia de hoy, existen en el programa de bachillerato los dos momentos antes sugeridos: en primero de bachillerato la asignatura se trata por áreas temáticas y en segundo se trata por autores como historia de la filosofía. En cuanto diseño curricular, encaja ahí adecuadamente la propuesta aquí presentada. Sin embargo, como se ha dicho antes, lo más importante es la "forma" como se implementan los dos momentos.

¿Qué herramientas proporcionadas por la asignatura de filosofía les quedan a los estudiantes para enfrentar los problemas filosóficamente? Para obtener una respuesta, sería interesante hacer un estudio entre la población adulta y preguntar qué recuerdan de la asignatura de filosofía. Solo podemos especular, pero las posibles respuestas serían principalmente de dos tipos, por un lado: "los griegos", "Aristóteles", "Kant", "Nietzsche”, "el mito de la caverna”, etc., básicamente el nombre de un autor; y por otro lado respuestas como: "el problema de si estamos predeterminados o somos libres", "los tipos de verdad", "las diversas teorías ontológicas sobre la realidad", "la tensión entre individuo y sociedad", "la relación entre ética y derecho", o mencionar dos o más problemas. ¿Cuál tipo de respuesta sería más recurrente? El resultado podría ser bastante revelador. Si adicionalmente se les preguntara qué le aporta la filosofía a la ciencia, podrían quizás contestar con la frase: "la filosofía es la madre de todas las ciencias"; o dirían una diferencia como que la ciencia es objetiva y comprobable y la filosofía es subjetiva y no comprobable; o que la filosofía se ocupa de lo que se escapa a la ciencia; o podrían mencionar un nombre como "Popper" o "Kuhn"; o simplemente no podrían señalar un aporte claro de la filosofía. No obstante, puede que sí en el otro sentido: que el heliocentrismo de Galileo contribuyó a que se replanteara el lugar del ser humano en el universo, que la evolución de Darwin aportó una nueva visión sobre qué es el ser humano, etc. Las respuestas serían muy dicientes sobre el enfoque que se está transmitiendo, aunque también cabría determinar si las respuestas se deben a la capacidad de memoria de cada individuo adulto o a una genuina huella filosófica en él.

De momento, según se ha venido proponiendo, comenzar la enseñanza por dar contenidos ya esquematizados y respuestas, no son modos instrumentales para incentivar a los estudiantes a comprender y experimentar para qué sirve la filosofía, por el contrario, restringen las habilidades filosóficas. A propósito, dice Mario Bunge (2006, 95): "El libro de texto tradicional facilita el 
facilismo, al no incluir problemas no resueltos. Lo mismo vale para los cursos en muchas facultades: no incluyen trabajos prácticos". Russell dijo algo muy similar: "Las clases magistrales como medio de instrucción son tradicionales en las universidades y fueron sin duda útiles antes de la invención de la imprenta" (Russell 1960). ${ }^{14}$ Este afirmó que muchas de estas tendencias de los encargados de la educación, que inhiben el desarrollo de la curiosidad natural y la creatividad de los estudiantes, se debe a un apego al pasado: "El deseo de preservar el pasado, en vez de la esperanza de crear el futuro, domina las mentes de aquellos que controlan la enseñanza de los jóvenes. La educación no debería apuntar a la conciencia muerta de hechos estáticos, sino a una actividad dirigida hacia el mundo que nuestros esfuerzos han de crear" (RusselL I9I6). ${ }^{15}$

Para innovar podría proponerse, muy tentativamente, un esquema de apropiación intelectual de la filosofía basado en el tratamiendo de problemas para aplicar al menos en seis pasos. Lo que proponemos es un aprendizaje de carácter heurístico con enseñanza "socratizada", en el sentido que se ha venido reiterando de que los profesores se encargarían de dar elementos y guía, pero poniendo al filosofante en el lugar central de la reflexión, dejando que mediante este esfuerzo dirigido se aproxime a los problemas de la filosofía y se apropie del "modo de ver" filosófico. Este esquema, y el enfoque propuesto en general, no tendrían que ser necesariamente implantados siempre en la totalidad de la asignatura, sino que podrían emplearse también puntualmente y dependiendo de los temas a tratar. La implementación del esquema supondría enseñar menos cantidad de contenidos al centrarse en la calidad del proceso de análisis, el cual cuesta mucho más tiempo y esfuerzo que leer un texto y responder un cuestionario sobre él; también supondría la elaboración de material autodidáctico totalmente nuevo. Los seis pasos, muy esquemáticamente, serían: 1. Entender en qué consiste el problema y por qué es importante; 2. Planear la resolución del problema; 3. Elaborar un primer intento de solución, o "aproximación", y ponerla a prueba; 4. Relacionar la solución propia con otros intentos de solución; 5. Analizar cuáles serían los efectos y las consecuencias de la implementación de las posturas en la realidad; y 6 . Determinar los interrogantes que queden por resolver y los nuevos problemas que se susciten en el análisis. Veamos con más detalle cada paso.

14 "Lectures as a means of instruction are traditional in universities and were no doubt useful before the invention of printing" (Russell 1960).

15 "The wish to preserve the past, rather than the hope of creating the future, dominates the minds of those who control the teaching of the young. Education should not aim at a dead awareness of static facts, but at an activity directed toward the world that our efforts are to create" (Russell i 9i6). 
1. Entender en qué consiste el problema y por qué es importante. Este punto les recalca a los estudiantes, de manera consciente o subconsciente, que las ideas y la realidad son siempre lo fundamental. Los problemas no deben ser entregados directamente sino suscitados a través de casos concretos y preguntas, porque forzar el problema sería quitar la oportunidad de aprender a darse cuenta. Hay que proporcionar recursos y orientación a los alumnos, pero partiendo de lo conocido por ellos para que su esfera de comprensión del mundo se vaya dilatando desde dentro, yendo de lo conocido a lo desconocido. Se cuenta con la destreza socrática de los docentes, es decir, la elección de las preguntas adecuadas y la entrega ingeniosa de las herramientas. Éstos deben dar solo los elementos justos de modo que los estudiantes puedan llevar a cabo el esfuerzo, como menciona Ferrater Mora $($ I 986,373$)$ hablando de un modelo de universidad que proponía y que llamaba la "Universidad-taller": "Cada tema lleva detrás de él, apoyándolo constantemente, el repertorio necesario, pero no más que el necesario, de conocimientos. No hay en las enseñanzas rigidez, pero tampoco desorden". Este paso se concreta con la formulación clara del problema, evitando al máximo la ambigüedad y los defectos lógicos.

2. Planear la resolución del problema. No deben contestar las preguntas enseguida ni el docente ni el estudiante. Se deben identificar los presupuestos iniciales, las motivaciones, los objetivos y las consecuencias esperadas de la solución. También se debe determinar qué tipo de problema es: empírico o conceptual, metodológico o de valoración, etc. Hay que corroborar que sea un problema filosófico y determinar si puede ser interdisciplinario, y en ese caso qué ciencias habría que tener en cuenta. En concordancia, se debe seleccionar el método adecuado, si hay varios métodos posibles elegir uno y si no hay ninguno, ingeniárselo; pueden propiciarse las situaciones para que ocurran los tres casos. Se debe ir simplificando y eliminanando lo que no es importante y ejercitar la capacidad analítica de descomponer el problema en partes más simples y reconstruirlo. Para inducir la ejecución de tales procesos, se pueden dar por ejemplo herramientas similares a la siguiente estructura lógica del relativismo moderado de los valores: " $a$ es valioso con respecto a $b$ por el organismo $c$ en la circunstancia $d$ con el fin $e$ y a la luz del cuerpo de conocimiento $f$ " (BUNGE I989, 14) y pedir que se analice la estructura misma y que se reemplacen las variables; en el cuerpo de conocimiento $f$ se puede incluir qué aporta la ciencia que sea relevante para resolver el problema concreto y de este modo comenzar a construir las conexiones transdisciplinarias. La operación inversa también se puede realizar en otras ocasiones: intentar formalizar un problema concreto.

3. Elaborar un primer intento de solución o "aproximación" y ponerla a prueba. Se comienza por formular lo más clara y definidamente posible las pro- 
puestas con sus argumentos. Luego se pasan a considerar las posibles objeciones a los propios argumentos e intentar solventarlas. Por último se concretan unas conclusiones parciales, que se entenderían como posturas aproximadas y que incluyen prever las consecuencias de la implementación de tales ideas. Esto le daría forma final al proceso que ha hecho el estudiante hasta el momento y éste lo valoraría como un proceso autónomo, independientemente de si después se da cuenta de que se había equivocado en la forma o en el planteamiento, o de que no había considerado muchas otras ideas. Adicionalmente, se corrigen los errores apuntados por el docente.

4. Relacionar la solución propia con otros intentos de solución. Aquí se introducen las propuestas de ideas de otros autores con gran maestría por parte del profesor, a ser posible no presentando de entrada las posturas a las que llegaron, sino reconstruyendo, en la medida de lo posible, el proceso por el cual los autores llegaron a sus conclusiones. De este modo, los estudiantes relacionarían tanto ese proceso como los resultados con los suyos propios y los analizarían con las herramientas que hayan ido adquiriendo y la dirección socratizada del profesor.

5. Analizar cuáles serían los efectos y las consecuencias de la aplicación de las posturas, del estudiante y de los autores, en la realidad, y su relación con los demás campos del saber.

6. Determinar los interrogantes que queden por responder y los nuevos problemas que se susciten en el análisis.

El anterior esquema coincide básicamente con la descripción del método científico en su forma más general como una secuencia de las siguientes operaciones cognitivas:

Identificar un problema — buscar información, métodos, instrumentos — intentar resolver el problema con la ayuda de esos medios - si es necesario inventar nuevos medios, producir nuevos datos o diseñar nuevos experimentos — derivar las consecuencias de tu solución (por ejemplo, predicciones) - comprobar las soluciones (por ejemplo, intentar reproducir tus hallazgos con medios alternativos) — corregir la solución si es necesario al repetir el ciclo — examinar el impacto de la solución sobre el cuerpo base de conocimiento, y establecer algunos de los nuevos problemas que hace surgir (Bunge y MAHER I996, 104) 16 $^{16}$

16 "Identify a problem — search for information, methods, instruments - try to solve the problem with the help of those means - if necessary invent new means, produce new data, or design new experiments - derive the consequences of your solution (e.g., predictions) - check the solutions (e.g., try to replicate your findings by alternative means) - correct the solution if necessary in repeating the cycle - examine the impact of the solution upon the body of background knowledge, and state some of the new problems it gives rise to" (BUNGE y MAHER I996, 104). 
Cabe preguntar en este punto si el método "socratizado" de enseñanza que aquí se propone es idéntico al método científico. La respuesta es que es un método muy similar, ciertamente en el carácter, como lo afirma Mario Bunge en La investigación cientifica (2000), obra que curiosamente fue traducida del inglés por el antes mencionado Manuel Sacristán:

El correcto planteamiento de los problemas filosóficos — su elección y su tratamiento - no difiere, o no debería diferir, demasiado del planteamiento correcto de los problemas científicos, por mucho que difieran los temas y las técnicas. Pero esto no es más que un modo ambiguo de decir que no hay más que un modo de plantear los problemas de conocimiento, ya sea en la ciencia pura, ya en la aplicada, ya en la filosofía: no se pueden plantear problemas de conocimiento sino científicamente. Esto puede ser dogmático, pero vale la pena intentarlo para ver si cambia la situación de la filosofía (BUNGE 2000, 191)

El esquema también está en consonancia con la conclusión a la que llegó Ferrater Mora en el antes mencionado "¿Para qué sirven los filósofos?”, donde dijo que la filosofía trata los problemas, que son problemas de todos y siempre urgentes, pero "sin excesiva prisa", en oposición a tratarlos de forma inmediata. Con este fin propuso dos enfoques: "Uno es darle a cualquier problema todas las vueltas necesarias para saber en qué consiste, incluyendo el averiguar si es verdaderamente un problema; el otro es proponer no una sola y única solución, sino varias" (Ferrater Mora 1994, 65). Por eso, como se ha visto, los puntos 1,2 y 3 del esquema de apropiación son los más extensos y primordiales. Pero, ¿por qué valdría la pena tratar los problemas urgentes con la calma que requiere el análisis filosófico? Ferrater prosiguió aclarando la utilidad: "El efecto es descorazonador para quienes piden soluciones inmediatas, pero puede ser, a la postre, beneficioso, sobre todo porque en no pocos casos las mejores soluciones a problemas que parecen urgentísimos son las que se ingenian a largo plazo" (Ferrater Mora i994, 65). Ferrater habló de calma y largo plazo, pero también de tener la intención de encontrar soluciones. La calma y el largo plazo, sin embargo, conllevan el peligro de acabar por no hacer nada, por eso los estudiantes deben ir proponiendo aproximaciones, aunque conscientes de que en principio se deben ver como temporales y siempre susceptibles de revisión.

Para que un esfuerzo de apropiación de la filosofía como el expuesto tenga más potencia, es conveniente que en el momento de enseñar diversidad de autores se incluyan filósofos iberoamericanos en el caso de Espańa y Latinoamérica. De este modo los estudiantes no asumirán que quienes saben hacer filosofía son siempre "otros": los alemanes, los estadounidenses o los franceses, por ejemplo, entendiendo que son unos simples espectadores y receptores 
de los que "sí saben pensar"; o lo que es peor, volverse indiferentes. También es muy relevante utilizar la propia lengua con confianza y deshacerse de otra creencia propagada, entre otros, por Martin Heidegger, quien apuntó cuando hablaba del término "ousía", que la historia de esta palabra básica de la filosofía era un ejemplo que demostraba que:

...la lengua griega es filosófica, es decir, no que el griego esté pleno de terminología filosófica, sino que filosofa en su estructura y formación básicas. Esto es cierto de cualquier lenguaje real, pero dependiendo en varios niveles. El nivel se mide por la profundidad y la fuerza del pueblo que habla la lengua y existe dentro de ella. Solo nuestra lengua alemana tiene el adecuado carácter filosófico profundo y creativo como los griegos (HeIDEGGER I994, $50 \mathrm{ff})^{17}$

Además de ideas de muchos filósofos famosos de que hay ciertas lenguas superiores, también existe la idea de que hay nacionalidades superiores, o un género superior para filosofar, entre otros. Con este tipo de afirmaciones el problema que impide la apropiación de la filosofía por parte de los estudiantes se acrecienta y podría denominarse "la doble barrera", a saber: primera barrera, la filosofía es de los filósofos; y segunda barrera, de los filósofos de ciertos países, que hablan ciertos idiomas y de cierto género. Incluso podría hablarse de una tercera barrera, y es que los estudiantes inclinados por la ciencia o la tecnología, pueden quedar totalmente desencantados si el tipo de filosofía que se les enseña dice que la filosofía y la ciencia chocan. El método de enseñanza por problemas, que posibilite la apropiación de la filosofía, sería, entre otros, una forma de derribar estas barreras y evitarle a los estudiantes la pérdida de tiempo que les supondría descubrir y derribar obstáculos que son tanto innecesarios como perjudiciales, porque causan atrofia filosófica en mayor o menor grado; y en el peor de los casos, desinterés por la filosofía al no sentirse partícipes de ella o indiferencia al no encontrarle un valor tangible.

17 “...die griechische Sprache philosophisch ist, d. h. nicht: mit philosophischer Terminologie durchsetzt, sondern als Sprache und Sprachgestaltung philosophierend. Das gilt von jeder echten Sprache, freilich in je verschiedenem Grade. Der Grad bemißt sich nach der Tiefe und Gewalt der Existenz des Volkes und Stammes, der die Sprache spricht und in ihr existiert. Den entsprechenden tiefen und schöpferischen philosophischen Charakter wie die griechische hat nur noch unsere deutsche Sprache" (HeIDEgGer I 994, $50 \mathrm{ff}$ ). 


\section{Conclusiones}

Partiendo de la pregunta por la utilidad de la asignatura de filosofía, hemos concluido que puede ser más útil cuando tiene una conexión estrecha con el saber científico, con el cual los estudiantes han estado familiarizados a través de todo su recorrido escolar. En afinidad con la metodología científica, se ha querido fundamentar el énfasis del aprendizaje en el tratamiento de problemas, como punto de partida más fecundo y provechoso para la evolución intelectual real del estudiante, quien sin dejar de estar guiado por el profesor haría un trabajo autónomo. Hemos considerado que siendo guiados de forma "socratizada", los estudiantes se apropiarían más fácilmente la filosofía como parte fundamental de su pensamiento y la aplicarían como recurso en todos los ámbitos de su vida, de modo que tenga un alcance personal, académico y social tan amplio y fértil como sea posible. Junto con la autonomía que estas herramientas generarían en los estudiantes, vendría la confianza en su propio pensamiento y la autoelaboración de un criterio personal fundamentado en la base sólida del análisis filosófico, no solo para pensar críticamente, sino también para aspirar a crear nuevos problemas y nuevo conocimiento según su vocación. Como resultado, se haría evidente que la filosofía les sería mucho más significativa y útil a ellos, y por ende a la comunidad educativa y a la sociedad. En definitiva, este replanteamiento de fondo busca que la filosofía como ejercicio intelectual y como modo de ver entre a formar parte permanente de la estructura de pensamiento de los estudiantes que pasen por esta asignatura. 


\section{BIBLIOGRAFÍA}

Bunge, M. I943, Temas de educación popular, Buenos Aires: El Ateneo.

Bunge, M. I967A, Foundations of Physics, Berlín: Springer-Verlag.

Bunge, M. I967B, Scientific Research, 2 v, Berlín: Springer-Verlag. Ed. rev.: (I 998)

Philosophy of Science. New Brunswick, NJ: Transaction.

Bunge, M. I977, Treatise on Basic Philosophy, vol. 3: The Furniture of the World,

Dordrecht: Reidel.

Bunge, M. I 979, Treatise on Basic Philosophy, vol. 4: A World of Systems, Dordrecht: Reidel.

Bunge, M. 1989, Treatise on Basic Philosophy, vol. 8: Ethics: The Good and the Right, Dordrecht: Reidel.

Bunge, M. 1998, Elogio de la curiosidad, Buenos Aires: Sudamericana.

Bunge, M. 2000, La investigación cientifica, México: Siglo veintiuno.

Bunge, M. 2003A, Philosophical Dictionary, Amherst: Phrometheus Books.

Bunge, M. 2003B, "Velocity operators and time-energy relations in relativistic quantum mechanics", International Journal of Theoretical Physics, 42: 135-42.

Bunge, M. 2006, 100 Ideas, Buenos Aires: Sudamericana.

Bunge, M. 2015, "Does the Aharonov-Bohm effect occur?", Foundations of Science, 20: 129-33.

Bunge, M. 20 17, Doing Science in the Light of Philosophy, World Scientific.

Bunge, M. y Maher, M. I 996, "Is Religious Education Compatible With Science

Education?”, Science \& Education, 5: 101-10.

Ferrater Mora, J. I974, Cambio de marcha en filosofía, Madrid: Alianza.

Ferrater Mora, J. I976, "La filosofía entre la ciencia y la ideología", Teorema, 6 (1): 27-42.

Ferrater Mora, J. I982, La filosofía actual, Madrid: Alianza.

Ferrater Mora, J. I986, Ventana al Mundo, Barcelona: Anthropos.

Ferrater Mora, J. I994, Mariposas y supercuerdas, Barcelona: Península.

Ferrater Mora, J. I998, De la materia a la razón, Madrid: Alianza.

Ferrater Mora, J. 2009, Diccionario de filosofía, vol. 4, Barcelona: Ariel.

Hare, W. 1987, "Russell's Contribution to Philosophy of Education", Russell: The Journal of Bertrand Russell Studies, [en línea] vol. 7: 25-41. Disponible en: https://escarpmentpress.org/russelljournal/article/view/1693/1719 [Consulta: 30 Ene. 2016]

Heidegger, M. I994, Vom Wesen der menschlichen Freiheit. Einleitung in die Philosophie (Sommersemester 1930), in: ders.: Gesamtausgabe, II. Abt.: Vorlesungen 1923-1944, Bd. 31, hg. v. H. Tietjen, 2. Aufl., Fráncfort: Vittorio Klostermann. 
Russell, B. 1916, "Education as a Political Institution", The Atlantic Monthly. [en línea] June 1916. Disponible en: http://www.theatlantic.com/magazine/ archive/1916/06/education-as-a-political-institution/305258/[Consulta: 30 Jun. 2016]

Russell, B. 1953, "Education's Place in a New Age", Saturday Night (Toronto), 68 , no. $22,1: 7-8$.

Russell, B. 1954, Education and the Good Life, Nueva York: Liveright Publishing. Russell, B. 1958, "The Divorce of Science and Culture", The Unesco Courier [en línea] 11, n. 2, (Feb. 1958): 4. Disponible en: http://unesdoc.unesco.org/ images/0006/000661/066121eo.pdf [Consulta: 30 Ene. 2016]

Russell, B. 1960, "The Great Intrusion: Democracy in Higher Education", Arkansas University Alumnus, XIII: 13-5.

Russell, B. 1967, Education and the Social Order, Londres: Routledge.

Russell, B. 2000, History of Western Philosophy, Londres: Routledge.

Russell, B. 2003, On Education, Londres: Routledge.

Russell, B 2009, The Basic Writings of Bertrand Russell, R. E. Egner y L. E. Denonn (ed.), Londres: Routledge.

SACRISTÁN, M. 1968, Sobre el lugar de la filosofía en los estudios superiores, Barcelona: Nova Terra. 